\title{
miR-218 inhibits the proliferation of human glioma cells through downregulation of Yin Yang 1
}

\author{
YONG GAO $^{1,2}$, LAISHENG SUN ${ }^{1}$, ZICHENG WU $^{1}$, CHENGMIN XUAN $^{3}$, JUNXIA ZHANG $^{2}$, \\ YONGPING YOU ${ }^{2}$ and XINCHENG CHEN ${ }^{1,2}$
}

\author{
${ }^{1}$ Department of Neurosurgery, Xinyi People's Hospital, Xinyi, Jiangsu 221400; ${ }^{2}$ Department of Neurosurgery, \\ The First Affiliated Hospital of Nanjing Medical University, Nanjing, Jiangsu 210029; ${ }^{3}$ Department of Hematology, \\ Xuzhou Children's Hospital, Xuzhou, Jiangsu 221006, P.R. China
}

Received August 31, 2016; Accepted May 18, 2017

DOI: $10.3892 / \mathrm{mmr} .2017 .8063$

\begin{abstract}
Malignant glioma is the most common cancer type of the nervous system and the mechanisms driving the occurrence and development remain unclear, preventing effective treatment of this disease. Therefore, novel and efficient therapies for glioma are required. MicroRNAs (miRNAs) are small non-coding RNAs that act as oncogenes or tumor suppressors in human cancer. In the present study, it was confirmed that Yin Yang-1 (YY1), a transcription factor that is part of the polycomb group protein $(\mathrm{PcG})$ family, is a direct target of miR-218 in human glioma cells. It was demonstrated that YY1 promoted glioma cell proliferation and miR-218 could inhibit glioma cell proliferation by targeting YY1, and indirectly reduced the degradation of p53. Together the results indicate that miR-218 functions as a tumor suppressor in human glioma and suggest that overexpression of miR-218 may be a potential strategy for the treatment of human glioma in the future.
\end{abstract}

\section{Introduction}

Gliomas are the most frequent types of primary tumor observed in the central nervous system (1). Despite progress in treatments, including surgery, radiation therapy, and chemotherapy $(2,3)$, the overall survival rate for patients suffering from glioma is among the lowest of all the main types of cancer and has not improved during recent decades (4). Thus, it is vitally important to discover an effective marker for early

Correspondence to: Dr Xincheng Chen, Department of Neurosurgery, Xinyi People's Hospital, 16 Renmin Road, Xinyi, Jiangsu 221400, P.R. China

E-mail: drxinchengchen@126.com

Professor Yongping You, Department of Neurosurgery, The First Affiliated Hospital of Nanjing Medical University, 300 Guangzhou Road, Nanjing, Jiangsu 210029, P.R. China

E-mail: dryongpingyou@126.com

Key words: miR-218, proliferation, YY1, glioma, p53 detection and as a target molecule for the development of glioma treatments.

MicroRNAs (miRNAs) are small, endogenous, noncoding RNAs that regulate gene transcription by complementary base pairing to specific mRNAs. miRNAs regulate a wide variety of biological processes including cell migration, invasion, proliferation, apoptosis, tumorigenesis and tissue morphogenesis (5-8). Numerous miRNAs have been reported to function in glioma development, including miR-132 (9), miR-503 (10), miR-661 (11), miR-16 (12), miR-21 (13), and miR-218 (14,15). Among these miRNA molecules, miR-218 has been demonstrated to be downregulated in low-grade glioma tissues and glioma cells compared with normal brain tissues (16). Previous studies have demonstrated that the overexpression of miR-218 contributes to not only the inhibition of proliferation, invasion and migration of glioma cells, however additionally the induction of apoptosis by downregulating transcription of miR-218 target genes, including inhibitor of nuclear factor $\kappa \mathrm{B}(\mathrm{NF}-\kappa \mathrm{B})$ kinase subunit $\beta(\mathrm{IKK}-\beta)$, lymphoid enhancer binding factor $1, \mathrm{NF}-\kappa \mathrm{B}$ and cyclin-dependent kinase 6 (CDK6) (14,17-19). However, the role of miR-218 in the regulation of glioma cell proliferation through targeting a transcription factor called Yin Yang-1 (YY1) remains to be fully elucidated.

YY1 is a universal and multifunctional zinc-finger transcription factor that can activate or repress a variety of genes (20). Increased expression of YY1 has been reported in prostate cancer, colon cancer, ovarian cancer and in breast cancer (21). By contrast, reduced expression of YY1 has been reported in types of melanoma, urothelial carcinomas and osteosarcomas (21). YY1 is constitutively elevated during the progression of brain gliomas compared with that of normal brain tissues, which shows a positive correlation with the progression of gliomas and meningiomas (22). However, at present there is no clear explanation about the role and regulated miRNAs of YY1 in gliomas. The current study aimed to elucidate the role of YY1 and miR-218 in human glioma cells and determine their regulatory association. It was demonstrated that YY1 was a promoting factor in glioma cell proliferation and miR-218 could inhibit glioma cell proliferation by targeting YY1. 


\section{Materials and methods}

Antibodies. YY1 antibodies were obtained from Santa Cruz (Santa Cruz Biotechnology, Inc., Dallas, TX, USA). Antibodies specific for p53 (cat. no. 2524) and $\beta$-actin (cat. no. 3700) were obtained from Cell Signaling Technology, Inc. (Danvers, MA, USA). Goat anti-mouse IgG (cat. no. AP124B) and goat anti-rabbit IgG (cat. no. AP307P) were purchased from EMD Millipore (Billerica, MA, USA).

Cell culture. Human glioma cells U251MG and 293T cells were obtained from the Cell Bank of Shanghai Institutes of Chinese Academy of Sciences (Shanghai, China). Cells were grown at $37^{\circ} \mathrm{C}$ with $5 \% \mathrm{CO}_{2}$ in DMEM medium (Invitrogen; Thermo Fisher Scientific, Inc., Waltham, MA, USA) containing $10 \%$ heat-inactivated fetal bovine serum (Evergreen Biological Engineering, Hangzhou, China).

miR-218 target prediction. The miR-218 target prediction was performed using bioinformatics algorithms from TargetScan (http://www.targetscan.org/) and PicTar (http://pictar.mdc-berlin.de/).

Constructs and production of lentivirus. For overexpression of miR-218, the miR-218 cDNA was inserted into the pGlV3/H1 plasmid (Shanghai GenePharma Co., Ltd., Shanghai, China) using BamHI and MluI sites. For silencing of miR-218, the short-hairpin RNA (shRNA) oligomer (target sequence: GATCCACATGGTTAGATCAAGCAC AACGATACATGGTTAGATCAAGCACAAACCGGTACA TGGTTAGATCAAGCACAATCACACATGGTTAGATCAA GCACAATTTTTTG) was annealed and then subcloned into the pGLV3/H1 plasmid by BamHI and MluI cloning sites. For knockdown of endogenous YY1, a specific shRNA oligomer (target sequence: CCTCCTGATTAT TCAGAATAT) was annealed and then introduced into the pLV-shRNA plasmid by BamHI and EcoRI cloning sites (23). For overexpression of YY1, the YY1 cDNA was inserted into the $3 \mathrm{X}$ Flag plasmid using EcoRI and XbaI sites. 3X Flag-p53 were obtained from Addgene, Inc. (Cambridge, MA, USA). Cells were transfected with Polyjet (SignaGen Laboratories, Gaithersburg, MD, USA) according to the manufacturer's protocol. The viruses were packaged in $293 \mathrm{~T}$ cells by cotransfecting the corresponding plasmids with the helper plasmids using Polyjet transfection reagent.

Reverse transcription-quantitative polymerase chain reaction $(R T-q P C R)$. RNA was extracted from stable lines using TRIzol reagent (Invitrogen; Thermo Fisher Scientific, Inc., Waltham, MA, USA) following manufacturers' instructions and $1 \mu \mathrm{g}$ total RNA was subjected for cDNA production using reverse transcription reagents (Roche Diagnostics $\mathrm{GmbH}$, Basel, Switzerland) according to the manufacturer's protocol. RT-qPCR reactions were performed with $3 \mu$ l template from each reaction with an ABI 7300 Real-Time PCR instrument (Applied Biosystems; Thermo Fisher Scientific, Inc.) using SYBR Green (Roche, Diagnostics GmbH). Primers for the amplification of YY1 and $\beta$-actin were as follows: YY1, forward CCTGGCATTGACCTCTCAGATCCA and reverse GGGCAAGCTATTGTTCTTGGAGCA; $\beta$-actin, forward
CATGTACGTTGCTATCCAGGC and reverse CGCTCG GTGAGGATCTTCATG. The products for YY1 and $\beta$-actin were 101 and $195 \mathrm{bp}$, respectively. PCR reaction mixture (10 $\mu \mathrm{M}$ forward and reverse primers, $10 \mu \mathrm{l}$ of LightCycler 480 SYBR Green I Master (Roche, Diagnostics GmbH), $3 \mu 1$ of cDNA template and $6 \mu$ of RNA-free water) was added into the reaction plate, and then run by PCR instrument. The following thermocycling conditions were used for the PCR: Initial denaturation at $95^{\circ} \mathrm{C}$ for $5 \mathrm{~min}$; followed by 40 cycles of $95^{\circ} \mathrm{C}$ for $15 \mathrm{sec}$ and $65^{\circ} \mathrm{C}$ for $35 \mathrm{sec}$. For each sample, the $\mathrm{C}_{\text {quantification }}$ cycle $(\mathrm{Cq})$ was determined and normalized to the average of the housekeeping gene $(\Delta \mathrm{Cq}=\mathrm{Cq}$ Unknown-Cq Housekeeping gene). The determination of gene transcript levels in each sample was performed using the $2^{-\Delta \Delta \mathrm{Cq}}$ method (24).

Luciferase reporter assay. The YY1 3'untranslated region (UTR)-Luc reporter was created by ligation of the YY1 3'UTR PCR product into the $S a c I$ and $X b a I$ site of the pmirGLO control vector (Shanghai GenePharma Co., Ltd., Shanghai, China). The mutant reporter was generated from pmirGLO-wild-type (WT)-YY1 3'UTR-Luc by deleting the binding site for miR-218 'UGAAUGU'. miR-21 promoter-containing (miPPR-21-containing) pmirGLO-basic plasmids were constructed (Shanghai GenePharma Co., Ltd.). Luciferase activity was measured using the Dual-Luciferase Reporter Assay System (Promega Corporation, Madison, WI, USA).

EdU assay. The proliferation of cells was evaluated by 5-ethynyl-2'-deoxyuridine (EdU) incorporation assay using an EdU assay kit (Guangzhou RiboBio Co., Ltd., China) according to the manufacturer's protocol. U251MG cell $\left(1 \times 10^{4}\right.$ cells per well) were incubated in triplicate in a 96-well plate for $48 \mathrm{~h}$ and then exposed to $50 \mu \mathrm{M}$ EdU for additional $2 \mathrm{~h}$ at $37^{\circ} \mathrm{C}$. Cells were fixed with $4 \%$ formaldehyde for $30 \mathrm{~min}$ at $25^{\circ} \mathrm{C}$ and $2 \mathrm{mg} / \mathrm{ml}$ glycine was added to neutralize the formaldehyde. Cells were then incubated with $0.5 \%$ Triton X-100 for $15 \mathrm{~min}$ at $25^{\circ} \mathrm{C}$ for permeabilization. After washing with PBS three times, the cells were treated with $100 \mu \mathrm{l}$ 1X Apollo Reaction Cocktail (Guangzhou RiboBio Co., Ltd.) for $30 \mathrm{~min}$. DNA was stained by Hoechst staining for $30 \mathrm{~min}$ to determine total number of cells and calculate percentage proliferation. The images were photographed under the Olympus IX-71 inverted microscope (Olympus Corporation, Tokyo, Japan).

Cell Counting Kit-8 (CCK-8) assay. A total of 5,000 cells in $100 \mu 1$ DMEM medium were plated in 96-well plates and grown under normal conditions. After cells had adhered to the plates, 2-(2-methoxy-4-nitrophenyl)-3-(4-nitrophenyl)-5(2,4-benzene disulfonate)-2H-tetrazolium monosodium salt (AAT Bioquest, Inc., Sunnyvale, CA, USA) was added into the medium and cells were incubated for $4 \mathrm{~h}$ at $37^{\circ} \mathrm{C}$. Living cells were counted daily by reading the absorbance at $450 \mathrm{~nm}$ using SynergyMx Multi-Mode Microplate Reader (Biotek Instruments, Inc., Winooski, VT, USA).

Colony formation assays. A total of 200 cells were plated in a 6-cm dish and incubated under normal conditions for 10 days. The cells were fixed with methanol and dyed with $0.05 \%$ crystal violet to assess colony staining. Following repeated 
washing with PBS, images were taken with a camera. Colonies containing more than 50 cells were counted.

Western blotting. Cells were lysed and equal amounts of cell lysates were subjected to $10 \%$ SDS-PAGE and then transferred onto polyvinylidene difluoride membranes (EMD Millipore, Billerica, MA, USA). Subsequent to blocking with 5\% skimmed milk, the membrane was incubated with primary antibodies [YY1 $(1: 1,000), \beta$-actin $(1: 1,000)]$ at $4^{\circ} \mathrm{C}$ overnight and then secondary antibodies $(1: 5,000)$ at room temperature for $1 \mathrm{~h}$. Bound antibodies were detected by the enhanced chemiluminescence Q6 system (Amersham Pharmacia Biotech, Piscataway, NJ, USA). Band densities were measured using ImageJ software 1.42q (National Institutes of Health, Bethesda, MD, USA). All examined gene expression levels were determined by normalizing the densitometry value of interest to that of the loading control.

Statistical analysis. SPSS software was used to perform statistical analyses. Data were presented as the mean \pm standard error. Statistical analyses were performed using SPSS, version 13.0 (SPSS, Inc., Chicago, IL, USA). Differences in multiple groups were determined by using one-way analysis of variance followed by Student-Newman-Keuls test. Comparison between two groups was performed by Student's t-test. $\mathrm{P}<0.05$ was considered to indicate a statistically significant difference.

\section{Results}

YY1 is a direct target of $m i R-218$. Bioinformatics analysis using TargetScan and PicTar algorithms indicated that YY1 is a hypothetical target gene of miR-218 (Fig. 1A). To further confirm the direct interaction between miR-218 and YY1 3'UTRs, plasmids containing WT (pmirGLO-WT-YY1 3'UTR) and mutant (MT)-type (pmirGLO-MT-YY1 3'UTR) YY1 3'UTRs were constructed. Luciferase reporter assays demonstrated that upregulation of miR-218 led to a notable decrease of luciferase activity of pmirGLO-WT-YY1 3'UTR in human glioma cells, without any significant change in luciferase activity of pmirGLO-MT-YY1 3'UTR (Fig. 1B). These results suggest that YY1 may be a direct target of miR-218 in human glioma cells.

YY1 promotes the proliferation of glioma cells. Whether YY1 participates in the proliferation of human glioma cells was investigated in vitro by overexpressing shRNA against YY1 to downregulate YY1 expression. The YY1-downregulated stable cell line was estabilshed by lentiviral infection and the infection efficiency was verified through the immunofluorescence images (Fig. 2A). The western blotting assay indicated that knockdown of YY1 expression could increase the expressions of p53 protein (Fig. 2B). Proliferation of glioma cells was examined by EdU, CCK-8 and colony formation experiments. Knocking down YY1 significantly decreased the proportion of EdU positive cells (Fig. 2C and D). CCK-8 experiments demonstrated that knocking down YY1 reduced the proliferation of glioma cells (Fig. 2E). The number of colonies was also reduced upon downregulating YY1 (Fig. 2F and G). Therefore, it was concluded that knocking down YY1 reduces the proliferation of glioma cells.
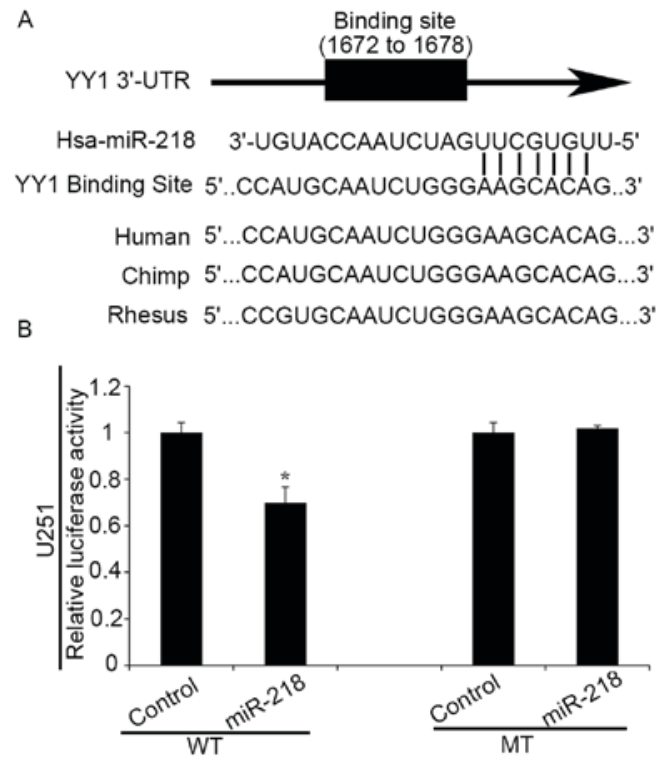

Figure 1. miR-218 directly targets YY1. (A) Putative binding sites of miR-218 within the YY1 3'UTR, as predicted by TargetScan and Pictar algorithms. (B) Luciferase reporter assay on the luciferase activity in WT YY1 3'UTR or MT-type YY1 3'UTR transfected U251MG cell. * $\mathrm{P}<0.05$ vs. control. miR, microRNA; YY1, Yin Yang-1; UTR, untranslated region; WT, wild-type; MT, mutant.

Downregulation of miR-218 promotes the proliferation of human glioma cells. Several studies have demonstrated that miR-218 has tumor-suppressive functions in human glioma $(14,15,18)$. However, the role of miR-218 in glioma remains unclear. Thus, the current study aimed to investigate the effects of miR-218 on proliferation by regulating YY1 in human glioma cells. miR-218 expression was first knocked down with an miRNA sponge specific for miR-218 (miR-218 sponge). Stable cell lines expressing the miR-218 sponge were constructed in U251MG cell by lentiviral infection (Fig. 3A). The infection efficiency of miR-218 was performed by monitoring the mRNA expression of YY1 (Fig. 3B). CCK-8 experiments demonstrated that knocking down miR-218 accelerated cell proliferation (Fig. 3C). Furthermore, colony formation ability was increased upon downregulation of miR-218 (Fig. 3D and E). Taken together, it was concluded that knocking down miR-218 induced the promotion of proliferation in glioma cells.

Overexpression of miR-218 inhibits the proliferation of glioma cells. To further explore the biological function of miR-218 in glioma cells, miR-218 was upregulated by lentiviral infection (Fig. 4A). The expression of YY1 mRNA was verified through RT-qPCR experiments and was observed to be significantly decreased in response to overexpressed miR-218 (Fig. 4B). Overexpression of miR-218 led to inhibition of glioma cell proliferation, as determined by CCK-8 (Fig. 4C) and colony formation experiments (Fig. 4D and E). Therefore, it was concluded that upregulating miR-218 inhibited the proliferation of human glioma cells.

miR-218 inhibits the proliferation of glioma cells through the YY1/p53 pathway. To clarify the potential mechanism of miR-218 in glioma cells, the YY1 and p53 expression in 


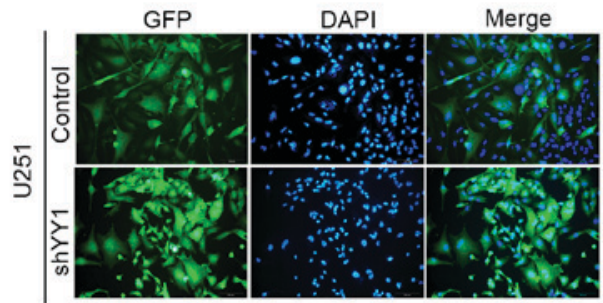

B

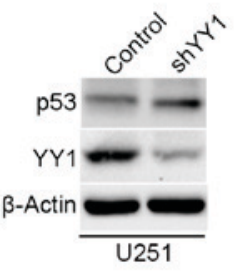

E

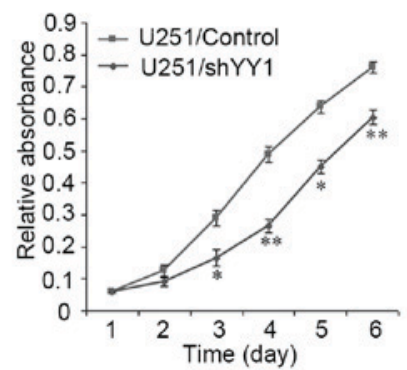

C

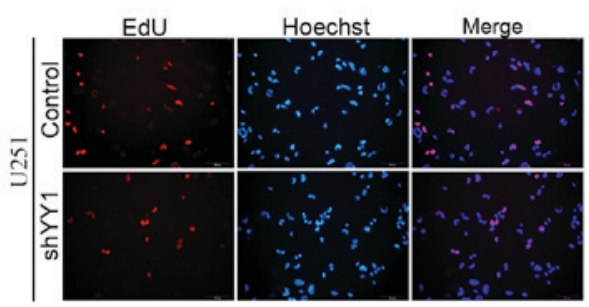

D

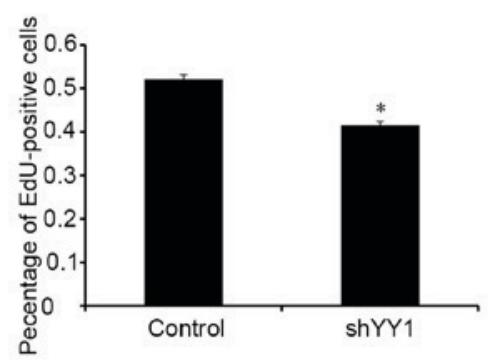

G

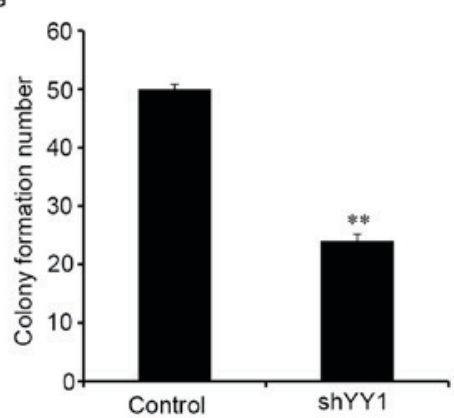

Figure 2. Silencing of YY1 significantly decreases glioma cell proliferation. (A) Control and shYY1 overexpressing lentivirus were packaged in 293T cells and used for infection of U251MG cell. The cells were dyed with DAPI to detect the infection rate. Scale bars, $100 \mu \mathrm{m}$. (B) Expression of YY1 in infected cells was analyzed by western blotting. (C) EdU analysis of cell proliferation. (D) Statistical analysis of the EdU experiment. (E) Cell Counting Kit-8 assay of cell viability. (F) Plate colony formation to analyze cell proliferation. (G) Statistics on the colony formation assay. ${ }^{*} \mathrm{P}<0.05$ and ${ }^{* *} \mathrm{P}<0.01$ vs. control. YY1, Yin Yang-1; sh, short hairpin; DAPI, 4',6-diamidino-2-phenylindole, dihydrochloride; EdU, 5-ethynyl-2'-deoxyuridine.

A

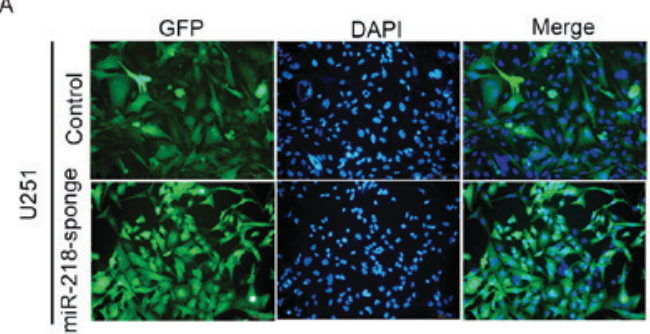

C

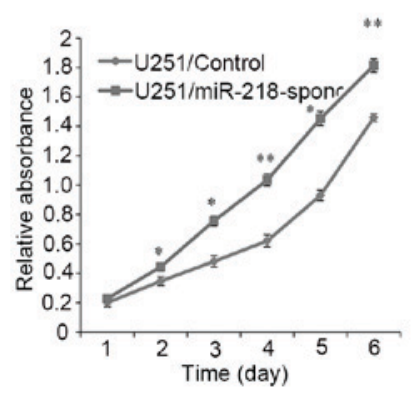

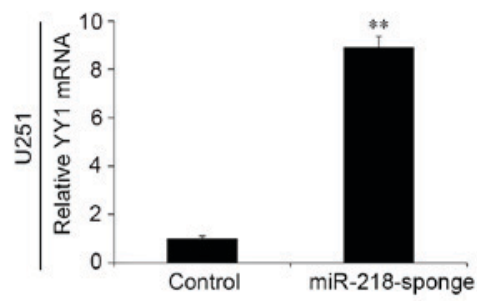

$\mathrm{E}$

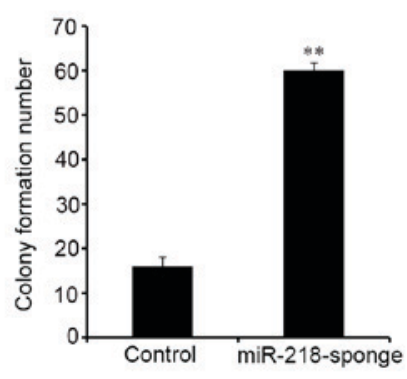

Figure 3. Downregulation of miR-218 promotes the proliferation of human glioma cells. (A) GFP images of stable lines overexpressing control and miR-218-sponge in U251MG cell. Scale bars, $100 \mu \mathrm{m}$. (B) YY1 mRNA level in control and miR-218-sponge expressing U251MG cell was quantified by reverse transcription-quantitative polymerase chain reaction. (C) Cell Counting Kit-8 assays on cell viability in control and miR-218-sponge expressing U251MG cell. (D) Plate colony formation assays on colony formation ability of control and miR-218-sponge expressing U251MG cell. (E) Statistical analysis of the colony formation assays. "P<0.05 and ${ }^{* *} \mathrm{P}<0.01$ vs. control. miR, microRNA; GFP, green fluorescent protein; YYI, Yin Yang-1; DAPI, 4',6-diamidino-2-phenylindole, dihydrochloride. 

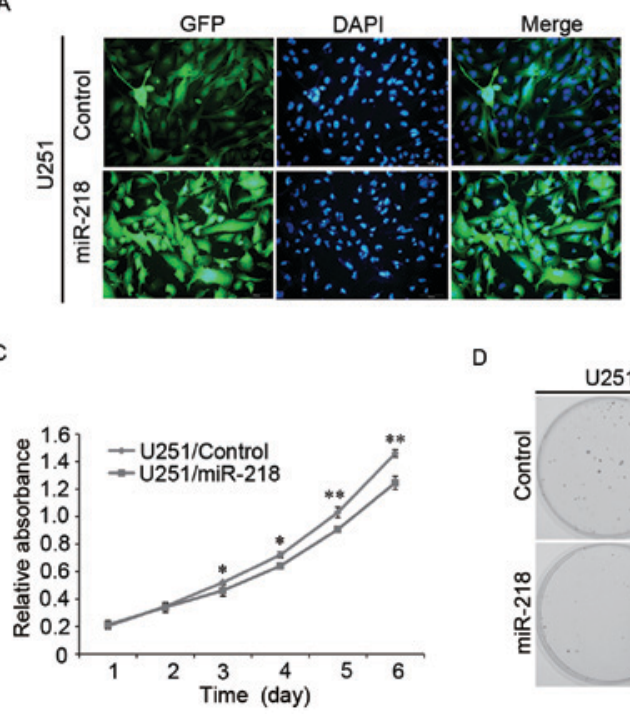

D

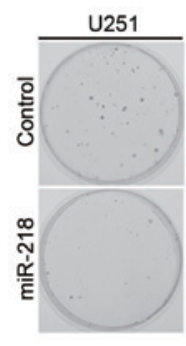

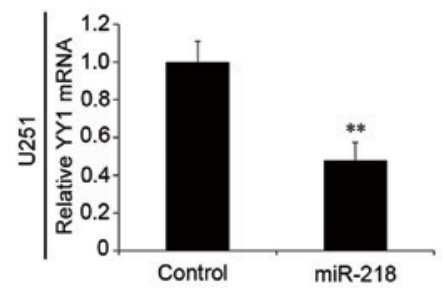

E

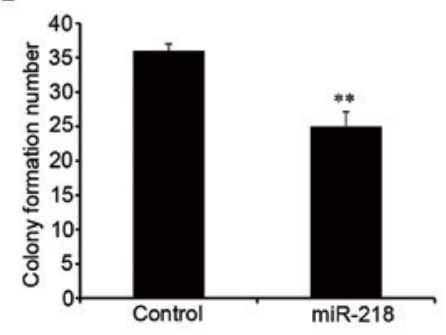

Figure 4. Overexpression of miR-218 significantly decreases the proliferation ability of glioma cells. (A) GFP images of the stable lines overexpressing control and miR-218 in U251MG cell. Scale bars, $100 \mu \mathrm{m}$. (B) YY1 mRNA was quantified by reverse transcription-quantitative polymerase chain reaction in control and miR-218 expressing U251MG cell. (C) Cell Counting Kit-8 assay of cell viability of control and miR-218 expressing U251MG cell. (D) Plate colony formation assays on colony formation ability of control and miR-218 expressing U251MG cell. (E) Statistical analysis of the colony formation assays. ${ }^{*}<<0.05$ and ${ }^{* *} \mathrm{P}<0.01$ vs. control. miR, microRNA; GFP, green fluorescent protein; YY1, Yin Yang-1; DAPI, 4',6-diamidino-2-phenylindole, dihydrochloride.

A

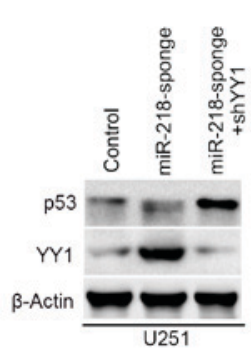

D
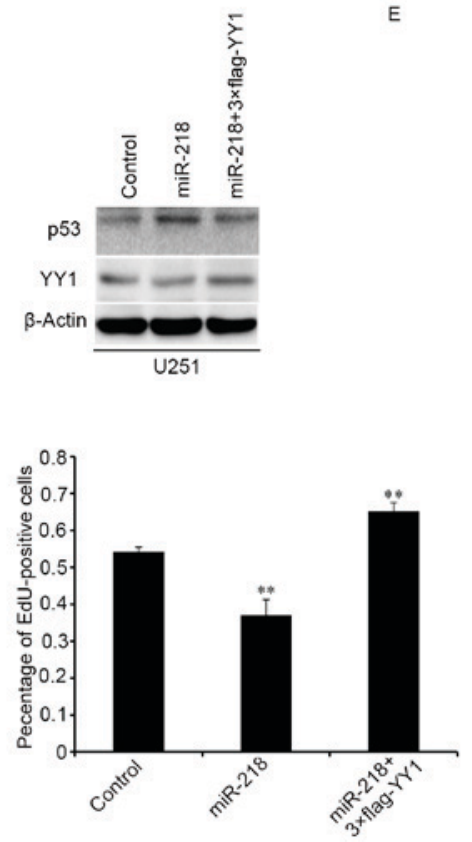

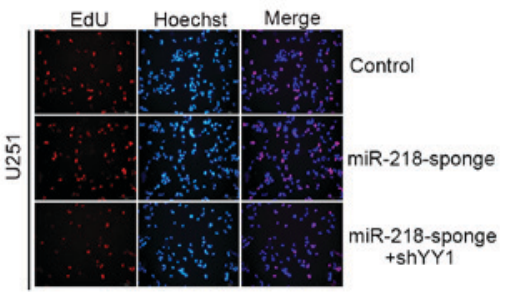

E

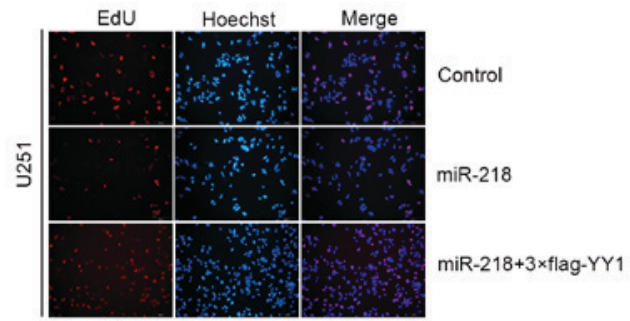

G

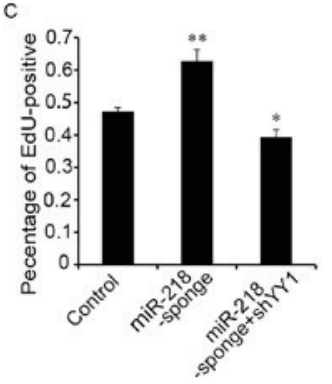

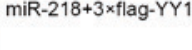

Figure 5. miR-218 mediated the proliferation ability of glioma cells by upregulation of YY1 and downregulation of p53 expression. (A) Western blotting results on the effects of miR-218 knock down and pLV-shYY1 and miR-218-sponge overexpressing on the protein expression level of YY1 and p53. (B) EdU analysis of cell proliferation in control, miR-218-sponge and miR-218-sponge + shYY1-expressing U251MG cell. (C) Statistical analysis of the EdU experiments. (D) Western blotting results on the effects of miR-218 upregulating and miR-218 and 3X Flag-YY1 overexpression on the protein expression levels of YY1 and p53. (E) EdU analysis on cell proliferation of control, miR-218 and mR-218 + 3X Flag-YY1 expressing U251MG cell. (F) Statistical analysis of the EdU experiments. (G) Western blotting identified YY1 and p53 expression following transfection with 3X Flag-p53 in U251MG cells. " $\mathrm{P}<0.05$ and ${ }^{* *} \mathrm{P}<0.01$ vs. control. miR, microRNA; YY1, Yin Yang-1; sh, short hairpin; EdU, 5-ethynyl-2'-deoxyuridine. 
sponge cells overexpressing miR-218 were detected. Western blot analysis demonstrated that downregulation of miR-218 upregulated the protein level of YY1, promoted the degradation of p53, and when we cotransfected the pLV-shYY1 and miR-218-sponge plasmids into U251MG, the expression levels of p53 were restored compared to upregulating miR-218-sponge (Fig. 5A). The results of EdU experiments and western blot analysis were consistent, and pLV-shYY1 could reverse the effect of miR-218-sponge in cell proliferation (Fig. 5B and C). Upregulating 3X Flag-YY1 and miR-218 led to similar results and the same protein expression trend with overexpressing pLV-shYY1 and miR-218-sponge, as determined by western blotting and EdU (Fig. 5D-F). However, when 3X Flag-p53 was overexpressed in U251MG cell, the expression of YY1 was not detected to be significantly different (Fig. 5G). Therefore, it was inferred that inhibition of glioma cell proliferation by overexpressing miR-218 in glioma cells may be mediated by downregulation of YY1 and upregulation of p53 expression which may be due to the reduction of $\mathrm{p} 53$ degradation by YY1.

Together the data indicated that YY1 is a direct target of miR-218 and serves a role in promoting the proliferation of glioma cells. The results suggest that miR-218 suppresses the proliferation of human glioma cells through the YY1/p53 pathway.

\section{Discussion}

The current study demonstrated that YY1 is a direct target of miR-218. To explore the exact role of miR-218 in glioma cells, miR-218 was overexpressed and silenced in human glioma cells by establishing stable cell lines using lentivirus. Silencing of miR-218 significantly promoted glioma cell proliferation by activating the YY1/p53 pathway, whereas overexpression of miR-218 inhibited glioma cell proliferation by suppressing the YY1/p53 pathway. It was concluded that post-transcriptional regulation of YY1 acts via miR-218 and that YY1/p53 signaling is an important mediator of the effects of miR-218 in glioma.

Previous studies have indicated that miR-218 is dramatically downregulated in human gliomas compared with normal brain tissues $(14,25,26)$. miR-218 has frequently been reported as a direct target of Bmil (15), IKK- $\beta$ (14), RTK (27), and CDK6 $(16,19)$ to prevent the proliferation, migration, invasion of glioma cells. To identify more mRNA targets of miR-218 in glioma cells, bioinformatics analysis was performed and it was identified that YY1 was a potential target of miR-218. The present study identified that miR-218 inhibited glioma cell proliferation by indirectly regulating p53 expression via directly targeting YY1. It is suggested that the miR-218-mediated inhibition of $\mathrm{YY} 1 / \mathrm{p} 53$ signaling pathway may be a promising method of treating glioma.

The transcription factor YY1 has been identified as a potential novel prognostic biomarker and therapeutic target. The role of YY1 in cancer is mediated by regulating several proteins in cancer development and progression including c-myc, c-fos, Erb-B2 receptor tyrosine kinase 2, and p53 (20,28). YY1 also interacts with numerous molecules that modulate cell proliferation and apoptosis, including p53, mouse double minute 2 homolog, enhancer of zeste homolog 2, caspases and histone deacetylases (20). YY1 is an important negative regulator of the tumor suppressor gene p53. Because of this, the level of p53 expression was detected. The current study exhibited that downregulation of YY1 inhibits the proliferation of glioma cells by increasing p53 expression. The data indicated that YY1 functions as a growth promoter of glioma cells by inhibiting tumor suppressor p53.

Taken together, the results suggested that miR-218 serves an important role in preventing the proliferation of glioma cells, and the results present a novel mechanism of miR-218-mediated direct suppression of the YY1/p53 pathway in glioma.

\section{Acknowledgements}

The present study was supported by the Foundation of Jiangsu Provincial Health Department (grant no. YG201514).

\section{References}

1. Davis FG and McCarthy BJ: Current epidemiological trends and surveillance issues in brain tumors. Expert Rev Anticancer Ther 1: 395-401, 2001

2. Buonerba C, Di Lorenzo G, Marinelli A, Federico P, Palmieri G, Imbimbo M, Conti P, Peluso G, De Placido S and Sampson JH: A comprehensive outlook on intracerebral therapy of malignant gliomas. Crit Rev Oncol Hematol 80: 54-68, 2011.

3. Sherman JH, Hoes K, Marcus J, Komotar RJ, Brennan CW and Gutin PH: Neurosurgery for brain tumors: Update on recent technical advances. Curr Neurol Neurosci Rep 11: 313-319, 2011.

4. Castro MG, Candolfi M, Kroeger K, King GD, Curtin JF, Yagiz K, Mineharu Y, Assi H, Wibowo M, Ghulam Muhammad AK, et al: Gene therapy and targeted toxins for glioma. Curr Gene Ther 11: 155-180, 2011.

5. Hwang HW and Mendell JT: MicroRNAs in cell proliferation, cell death, and tumorigenesis. Br J Cancer 94: 776-780, 2006.

6. Gabriely G, Wurdinger T, Kesari S, Esau CC, Burchard J, Linsley PS and Krichevsky AM: MicroRNA 21 promotes glioma invasion by targeting matrix metalloproteinase regulators. Mol Cell Biol 28: 5369-5380, 2008.

7. Kloosterman WP and Plasterk RH: The diverse functions of microRNAs in animal development and disease. Dev Cell 11: 441-450, 2006.

8. Yang L, Li Q, Wang Q, Jiang Z and Zhang L: Silencing of miRNA-218 promotes migration and invasion of breast cancer via Slit2-Robol pathway. Biomed Pharmacother 66: 535-540, 2012.

9. Wang H, Li XT, Wu C, Wu ZW, Li YY, Yang TQ, Chen GL, Xie XS, Huang YL, Du ZW and Zhou YX: miR-132 can inhibit glioma cells invasion and migration by target MMP16 in vitro. Onco Targets Ther 8: 3211-3218, 2015.

10. Liu H, Song Z, Liao D, Zhang T, Liu F, Zheng W, Luo K and Yang L: miR-503 inhibits cell proliferation and invasion in glioma by targeting L1CAM. Int J Clin Exp Med 8: 18441-18447, 2015.

11. Li Z, Liu YH, Diao HY, Ma J and Yao YL: miR-661 inhibits glioma cell proliferation, migration and invasion by targeting hTERT. Biochem Biophys Res Commun 468: 870-876, 2015.

12. Han J and Chen Q: miR-16 modulate temozolomide resistance by regulating BCL-2 in human glioma cells. Int J Clin Exp Pathol 8: 12698-12707, 2015.

13. Costa PM, Cardoso AL, Custódia C, Cunha P, Pereira de Almeida L and Pedroso de Lima MC: miRNA-21 silencing mediated by tumor-targeted nanoparticles combined with sunitinib: A new multimodal gene therapy approach for glioblastoma. J Control Release 207: 31-39, 2015

14. Song L, Huang Q, Chen K, Liu L, Lin C, Dai T, Yu C, Wu Z and Li J: miR-218 inhibits the invasive ability of glioma cells by direct downregulation of IKK- $\beta$. Biochem Biophys Res Commun 402: 135-140, 2010.

15. Tu Y, Gao X, Li G, Fu H, Cui D, Liu H, Jin W and Zhang Y: MicroRNA-218 inhibits glioma invasion, migration, proliferation, and cancer stem-like cell self-renewal by targeting the polycomb group gene Bmi1. Cancer Res 73: 6046-6055, 2013.

16. Jun GJ, Zhong GG and Ming ZS: miR-218 inhibits the proliferation of glioma U87 cells through the inactivation of the CDK6/cyclin D1/p21 pathway. Oncol Lett 9: 2743-2749, 2015. 
17. Liu Y, Yan W, Zhang W, Chen L, You G, Bao Z, Wang Y, Wang H, Kang $C$ and Jiang T: miR-218 reverses high invasiveness of glioblastoma cells by targeting the oncogenic transcription factor LEF1. Oncol Rep 28: 1013-1021, 2012.

18. Xia H, Yan Y, Hu M, Wang Y, Wang Y, Dai Y, Chen J, Di G Chen $\mathrm{X}$ and Jiang X: miR-218 sensitizes glioma cells to apoptosis and inhibits tumorigenicity by regulating ECOP-mediated suppression of NF- $\kappa$ B activity. Neuro Oncol 15: 413-422, 2013.

19. Zhang JM, Sun CY, Yu SZ, Wang Q, An TL, Li YY, Kong YL and Wen YJ: Relationship between miR-218 and CDK6 expression and their biological impact on glioma cell proliferation and apoptosis. Zhonghua Bing Li Xue Za Zhi 40: 454-459, 2011 (In Chinese).

20. Gordon S, Akopyan G, Garban H and Bonavida B: Transcription factor YY1: Structure, function, and therapeutic implications in cancer biology. Oncogene 25: 1125-1142, 2006.

21. Kashyap V and Bonavida B: Role of YY1 in the pathogenesis of prostate cancer and correlation with bioinformatic data sets of gene expression. Genes Cancer 5: 71-83, 2014.

22. Baritaki S, Chatzinikola AM, Vakis AF, Soulitzis N Karabetsos DA, Neonakis I, Bonavida B and Spandidos DA: YY1 Over-expression in human brain gliomas and meningiomas correlates with TGF-beta1, IGF-1 and FGF-2 mRNA levels. Cancer Invest 27: 184-192, 2009.
23. Liao WR, Hsieh RH, Hsu KW, Wu MZ, Tseng MJ, Mai RT, Wu Lee YH and Yeh TS: The CBF1-independent Notch1 signal pathway activates human c-myc expression partially via transcription factor YY1. Carcinogenesis 28: 1867-1876, 2007.

24. Livak KJ and Schmittgen TD: Analysis of relative gene expression data using real-time quantitative PCR and the 2(-Delta Delta C(T)) method. Methods 25: 402-408, 2001.

25. Setty M, Helmy K, Khan AA, Silber J, Arvey A, Neezen F, Agius P, Huse JT, Holland EC and Leslie CS: Inferring transcriptional and microRNA-mediated regulatory programs in glioblastoma. Mol Syst Biol 8: 605, 2012.

26. Skalsky RL and Cullen BR: Reduced expression of brain-enriched microRNAs in glioblastomas permits targeted regulation of a cell death gene. PLoS One 6: e24248, 2011.

27. Mathew LK, Skuli N, Mucaj V, Lee SS, Zinn PO, Sathyan P, Imtiyaz HZ, Zhang Z, Davuluri RV, Rao S, et al: miR-218 opposes a critical RTK-HIF pathway in mesenchymal glioblastoma. Proc Natl Acad Sci USA 111: 291-296, 2014.

28. Sui G, Affar el B, Shi Y, Brignone C, Wall NR, Yin P, Donohoe M, Luke MP, Calvo D, Grossman SR and Shi Y: Yin Yang 1 is a negative regulator of p53. Cell 117: 859-872, 2004. 